\title{
Intentional Replantation for a Right Mandibular Premolar
}

\author{
Sheng-Jin Xue ${ }^{1}$, Qing-Bin Zhang ${ }^{2}$ and Wei-Qun Guan ${ }^{1}$ \\ ${ }^{1}$ Department of Stomatology, Fujian Medical University Union Hospital, Fuzhou, China \\ ${ }^{2}$ Department of Stomatology, Xiamen Humanity Hospital, Affiliated to Fujian Medical University, Xiamen, China
}

\begin{abstract}
The present study reports a case of intentional replantation (IR) for a right mandibular second premolar (\#45). For the present case, root canal retreatment was first considered after removal of the metal post and core. When the metal post and core could not be removed from \#45, micro-apical surgery or intentional tooth replantation was performed. Six-month postoperative evaluation revealed that the right mandibular second premolar had no obvious symptoms of discomfort, and the clinical follow-up revealed uneventful healing and good bone regeneration. The short-term clinical efficacy was acceptable. For cases with root canal treatment failure, when the apical surgical access could not be established due to the adjacent important anatomical structures, IR may be employed as an accepted endodontic treatment procedure.
\end{abstract}

Key Words: Root canal treatment failure, Post-core crown, Root canal retreatment, Intentional replantation.

How to cite this article: Xue SJ, Zhang QB, Guan WQ. Intentional Replantation for a Right Mandibular Premolar. J Coll Physicians Surg Pak 2022; 32(02):253-255.

\section{INTRODUCTION}

Intentional replantation (IR) refers to a clinical technique for extracting difficult teeth and implanting the same into the alveolar socketafterthorough treatmentunderdirect vision in vitro. ${ }^{1}$ Nowadays, cases with root canal treatment failure are mostly treated by microscopic apical surgery. However, for cases undergoing apical surgery, this remains difficult to implement, or the results lead to imperfect outcomes. The present study reports a case of IR for a right mandibular second premolar.

\section{CASE REPORT}

A 25-year female patient was referred for the management of tooth \#45 (Figure 1a). The radiograph revealed incomplete root filling, with a high density post located in the upper $1 / 3$ rd of the root canal and a low density shadow in the apical region (Figure $2 \mathrm{a})$. The cone beam computed tomography (CBCT) revealed underfilling of the \# 45 root canals at $2.8 \mathrm{~mm}$ (Figure $2 \mathrm{~b}$ ).

The diameter of the periapical affected area was approximately $5.0 \mathrm{~mm}$ (Figure $2 \mathrm{c}$ ), and the distance from the apex to the mental nerve was approximately $2.4 \mathrm{~mm}$. The thickness of the residual dentin wall was approximately $2 \mathrm{~mm}$ (Figure $2 \mathrm{~d}$ ). Ultrasound oscillation was used to remove the metal posts and cores, butthe procedure failed (Figure 1b). Hence, IR was planned.

Correspondence to: Wei-Qun Guan, Department of Stomatology, Fujian Medical University Union Hospital, Fuzhou, China

E-mail: guanwq95314@163.com

Received: January 01, 2020; Revised: March 21, 2020;

Accepted: March 23, 2020

DOI: https://doi.org/10.29271/jcpsp.2022.02.253

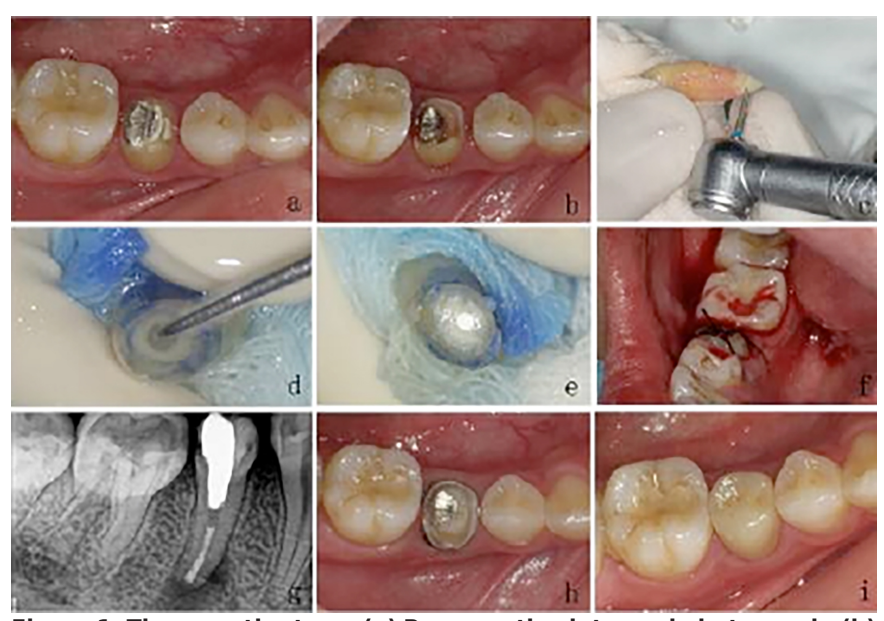

Figure 1: Therapeutic steps: (a) Preoperative intraoral photograph, (b) The post and core cannot be removed, (c) Apicoectomy, (d) Retrograde preparation, (e) iRootBP retrogradefilling, (f) Resetand suture, (g) Radiographic examination right after tooth replantion, (h) Dental preparation, (i) Dental preparation.

The granulation tissue was carefully removed from the tooth and fragments. Later, $3 \mathrm{~mm}$ of the root tip was resected perpendicular to the long axis of the root to reduce $98 \%$ of the apical ramifications and $93 \%$ of the lateral canals (Figure 1c). Afterwards, retrograde preparation of the root canal was extra-orally performed (Figure 1d). Root-end filling was performed with iRoot BP on the canal (Figure 1e). Then, the tooth was replanted in the alveolar socket in its original position, and stabilised, using a figure-of-eight suture (Figure 1f). The postoperative radiograph revealed a $3-\mathrm{mm}$ root-end filling coaxial to the canal (Figure 1g). Postoperative instructions were given, which included soft diet for one week and careful brushing after every meal. In addition, $100 \mathrm{mg}$ of doxycycline, BID, was prescribed for seven days. The suture was removed after two weeks. 
The patient reported no postoperative pain after treatment, and the tooth was firm and healthy after three months of follow-up. After crown installation, the patient was able to eat and chew normally (Figures $1 \mathrm{~h}$ and $1 \mathrm{i}$ ). The six-month postoperative evaluation revealed that the right mandibular second premolar had no obvious symptoms of discomfort (Figures 3a-3c).
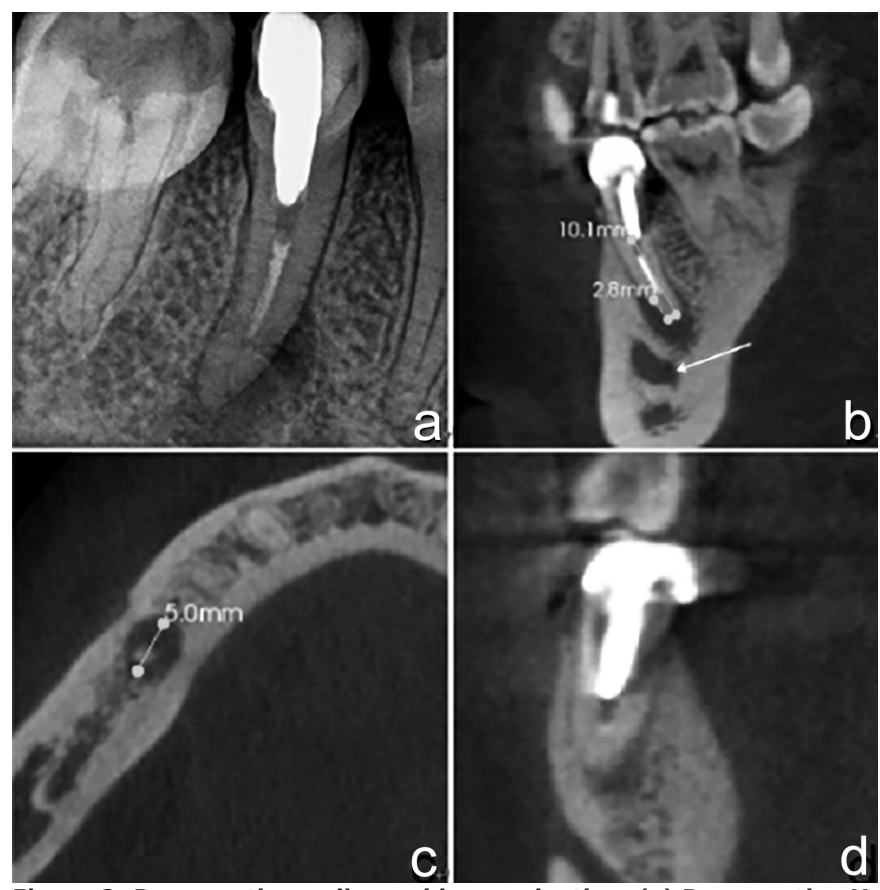

Figure 2: Preoperative radiographic examination: (a) Preoperative $\mathrm{X}$ ray, (b) Coronal plane of CBCT, (c) Horizontal plane of CBCT, (d) Sagittal plane of СВCT.
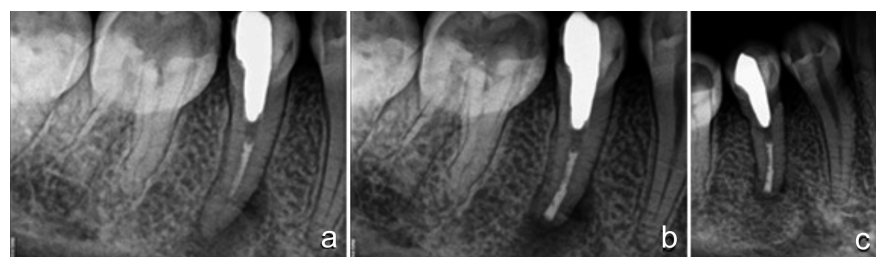

Figure 3: Preoperative and postoperative radiographic examination: (a)preoperative radiographic examination, (b) radiographic examination right after tooth replantation, (c) postoperative radiographic examination after 6 months.

\section{DISCUSSION}

IR is a useful technique for the treatment of difficult cases. ${ }^{2-4}$ In this study, there was no occlusion between the \#45 tooth and jaw teeth, there was no need for periodontal fixation after implantation of the alveolar fossa, and merely a single-filament suture was used to fix it. IR has its unique advantages in some difficult cases. Lu etal. reported that the treatment of refractory periapical periodontitis of the mandibular second molar by IR has a good clinical effect. ${ }^{5}$ With improved clinical procedures, based on the understanding of prognostic factors, IR can be a feasible treatment option for treating teeth with a C-shaped canal. In a study of 41 cases, followed up for 11 years, the fouryear success rate was $83.4 \%$, and the 11 -year success rate was $73.0 \%$. ${ }^{6}$ Periodontal maintenance after IR can effectively reduce secondary occlusal trauma and maintain normal perio- dontal tissue function.

The preservation rate of severe periodontitis teeth in 5-12 years is $88.2 \%{ }^{7}$ Asgary et al. reported that intentional tooth replantation can achieve good clinical results in the treatment of pulpal complications. ${ }^{8}$ The success rate of a tooth replantation is $>90 \%$. A meta-analysis revealed that IR was more cost-effective, when compared to implants. Hence, this can be used as a feasible treatment before implantation. ${ }^{9}$ Since the clinical follow-up was short in the present study, further studies with longerfollow-ups should be conducted.

In conclusion, for cases with root canal treatment failure, when the apical surgical access could not be established due to an adjacent important anatomical structure, IR may be employed as an acceptable endodontic treatment procedure.

\section{PATIENT'S CONSENT:}

This study was conducted in accordance with the Declaration of Helsinki. The patient signed a document of informed consent.

\section{CONFLICT OF INTEREST:}

The authors declared no conflict of interest.

\section{AUTHORS' CONTRIBUTION:}

SJX, QBZ, WQG: Conceived the idea and conceptualised the study, collected the data, analysed the data, drafted and reviewed the manuscript.

All authors read and approved the final draft.

\section{REFERENCES}

1. Cho SY, Lee SJ, Kim E. Clinical outcomes after intentional replantation of periodontally involved teeth. J Endod 2017; 43(4):550-5. doi: 10.1016/j.joen.2016.11.024.

2. Becker BD. Intentional replantation techniques: A critical review. J Endod 2018; 44(1):14-21. doi: 10.1016/j. joen.2017.08.002.

3. Nagappa G, Aspalli S, Devanoorkar A, Shetty S, Parab P. Intentional replantation of periodontally compromised hopeless tooth. J Indian Soc Periodontol 2013; 17(5): 665-9. doi:

4. Grzanich D, Rizzo G, Silva RM. Saving natural teeth: Intentional replantation protocol and case series. J Endod 2017; 43(12):2119-24. doi: 10.1016/j.joen.2017. 08.009 .

5. Lu WY, Pan J, Chen L. Intentional replantation for treatment of a mandibular second molar with persistent chronic periapical periodontitis: A case report. Zhonghua Kou Qiang Yi Xue Za Zhi 2018; 53(7):484-5. doi: 10.3760/cma. j.issn.1002-0098.2018.07.011.

6. Jang Y, Lee SJ, Yoon TC, Roh BD, Kim E. Survival rate of teeth with a c-shaped canal after intentional replantation: A study of 41 cases for up to 11 Years. J Endod 2016; 42(9):1320-5. doi: 10.1016/j.joen.2016.05.010.

7. Hou GL, Hou LT, Weisgold A. Survival rate of teeth with periodontally hopeless prognosis after therapies with intentional replantation and perioprosthetic procedures - a study of case series for 5-12years. Clin Exp Dent Res 2016; 2(2):85-95. doi: 10.1002/cre2.25. 
8. Asgary S, Talebzadeh B. Intentional replantation of a molar with several endodontic complications. J Stomatol Oral Maxillofac Surg 2019; 120(5):489-92. doi: 10.1016/j. jormas.2018.11.019.
9. Mainkar A. Systematic Review of the survival of teeth intentionally replanted with a modern technique and costeffectiveness compared with single-tooth implants. J Endod 2017; 43(12):1963-8. doi: 10.1016/j.joen.2017. 08.019 . 